This item was submitted to Loughborough's Research Repository by the author.

Items in Figshare are protected by copyright, with all rights reserved, unless otherwise indicated.

\title{
A parameter identifying a Kalman filter observer for vehicle handling dynamics
}

PLEASE CITE THE PUBLISHED VERSION

PUBLISHER

Professional Engineering Publishing / @ IMechE

VERSION

VoR (Version of Record)

LICENCE

CC BY-NC-ND 4.0

\section{REPOSITORY RECORD}

Hodgson, G., and Matt C. Best. 2009. "A Parameter Identifying a Kalman Filter Observer for Vehicle Handling Dynamics". figshare. https://hdl.handle.net/2134/5110. 
This item was submitted to Loughborough's Institutional Repository (https://dspace.lboro.ac.uk/) by the author and is made available under the following Creative Commons Licence conditions.

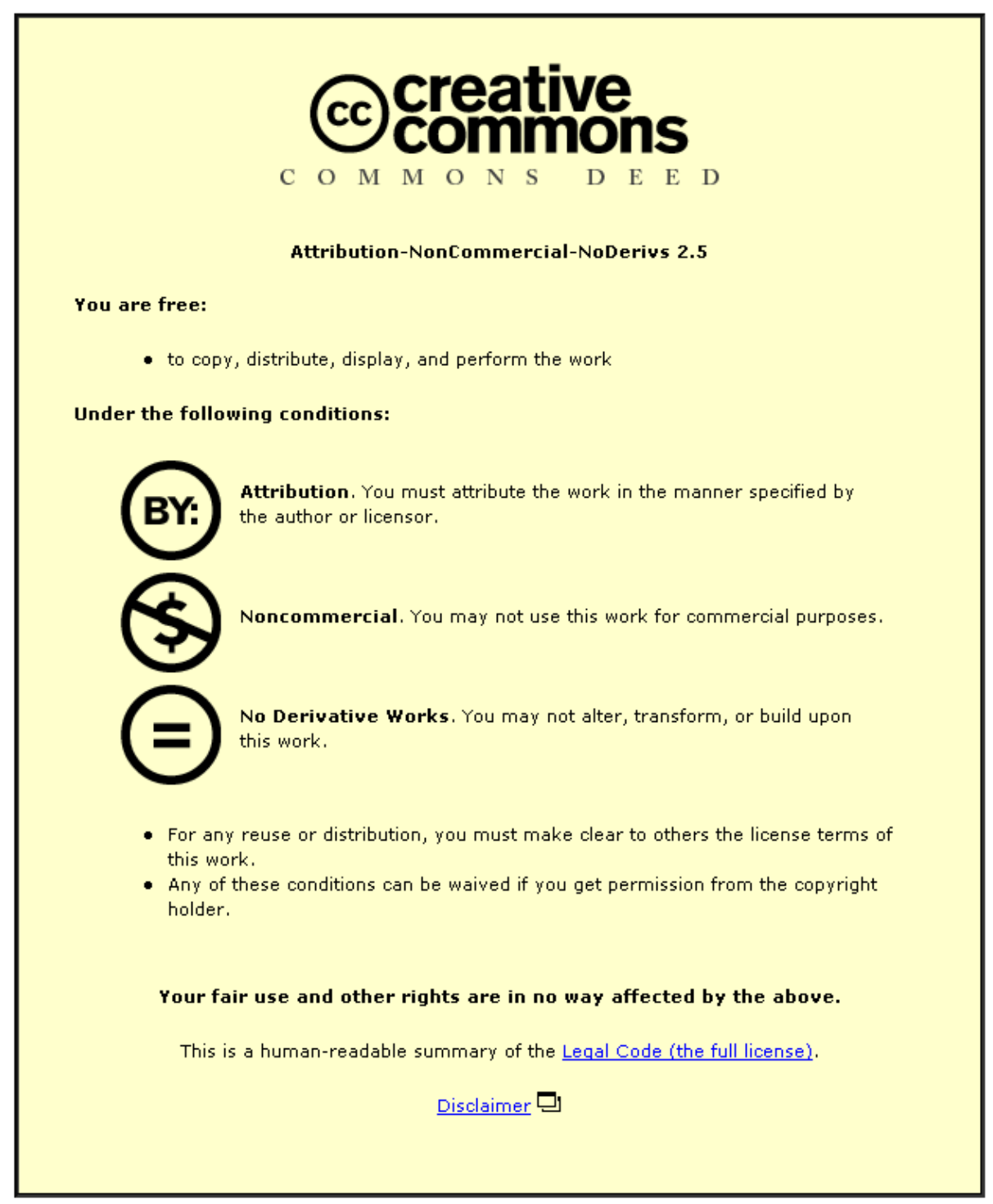

For the full text of this licence, please go to: http://creativecommons.org/licenses/by-nc-nd/2.5/ 


\title{
A parameter identifying a Kalman filter observer for vehicle handling dynamics
}

\author{
G Hodgson and M C Best* \\ Department of Aeronautical and Automotive Engineering, Loughborough University, Loughborough, Leicestershire, UK
}

The manuscript was received on 22 September 2004 and was accepted after revision for publication on 10 April 2006.

DOI: 10.1243/09544070D18304

\begin{abstract}
The paper presents a method for designing a non-linear (i.e. extended) Kalman filter that is also parameter adaptive and hence capable of online identification of its model. The filter model is deliberately simple in structure and low order, yet includes non-linear, load-varying tyre force calculations to ensure accuracy over a range of test conditions. Shape parameters within the (Pacejka) tyre model are adapted rapidly in real time, to maintain excellent state reconstruction accuracy, and provide valuable real-time lateral and vertical tyre force information. The filter is tested in both simulated and test vehicle environments and provides good results. The paper also provides an illustration of the importance of good Kalman filter design practice in terms of selection and tuning of the noise matrices, particularly in terms of the influence of model/sensor error cross-correlations.
\end{abstract}

Keywords: handling dynamics, Kalman filter, parameter estimation, state observer

\section{INTRODUCTION}

Kalman filters in vehicle dynamics have become popular in two areas. Initially as state observers, based around traditionally installed sensors, and more recently as a way of generating yaw information from the increasingly popular global positioning system (GPS) installations.

High-order models within Kalman filters allow for estimation of a number of vehicle dynamic states including wheel speeds and roll and yaw rates. Ray $[\mathbf{1}, \mathbf{2}]$ examines the procedure of using a Kalman filter with a large sensor set to estimate the road friction, and also regresses a slip versus tyre force characteristic. Zuurbier et al. [3] presents a similar concept, using a different sensor set.

More recent work focuses on the use of Kalman filters with GPS. There are two trends emerging: using Kalman filters to estimate the vehicle yaw angle (heading angle) along with the bias [4-6], and then using Kalman filters to estimate vehicle states [7].

The potential for Kalman filters to be used as observers for vehicle dynamics control has also led

\footnotetext{
* Corresponding author: Department of Aeronautical and Automotive Engineering, and Transport Studies, Loughborough University, Ashby Road, Loughborough, Leicestershire LE11 3TU, UK.email: m.c.best@lboro.ac.uk
}

to research on the robustness of these systems. Huh et al. [8] present a method of maintaining well conditioned matrices by scaling of the states and sensors within the Kalman filter, alongside a model error compensator technique.

Further potential for vehicle handling dynamics control through the use of Kalman filters is demonstrated by Best et al. [9] who adapt tyre parameters in a linear model. The concept of adaptation of parameters could be further expanded in future to fit into an integrated vehicle dynamics control system that controls not only the handling dynamics but also the vertical and driveline dynamics. With a combination of online adaptation and suitably matched control theory, key vehicle states and time-varying parameters could be controlled with a reduced, and thus cheaper, sensor set.

The aim of the present paper is to extend the earlier work of Best et al. [9] towards a practically viable, simple, yet adaptive observer. The key component is the choice of model within the filter; this should be low order to ensure practicality in the number of sensors required, and to allow stable, yet rapid online parameter identification. However, it must also be non-linear to ensure sufficient accuracy across a range of operating conditions. Good model accuracy is an essential prerequisite of any well balanced Kalman filter (i.e. one that achieves 
approximately equal balance between its reliance on the model and sensor information sources).

The paper starts by discussing the trade-off between model and sensor information, and by illustrating the importance of good design practice, in section 2. The identifying Kalman filter design is then described in section 3, and this is demonstrated in simulation in section 4 , and on vehicle test data in section 5 .

\section{CROSS-CORRELATION MATRIX}

This section considers the importance of good design practice for Kalman filters, particularly those designed for systems with sensor modelling errors. The design of a linear time-invariant continuous Kalman filter is adequate to illustrate the issues.

Consider a state-space system with model and system errors $\omega$ and $v$ respectively

$$
\begin{aligned}
& \overline{\dot{\boldsymbol{x}}}=\mathrm{A} \overline{\boldsymbol{x}}+\mathrm{B} \boldsymbol{u}+\boldsymbol{\omega} \\
& \boldsymbol{y}_{\mathrm{s}}=\mathrm{C} \overline{\boldsymbol{x}}+\mathrm{D} \boldsymbol{u}+\boldsymbol{v}
\end{aligned}
$$

where $\overline{\boldsymbol{x}}$ represents the true state of the physical system, $\omega$ describes the error induced by describing the state differential using the linear model A, B. and $v$ describes the error between the sensor measurement $\boldsymbol{y}_{\mathrm{s}}$ and the linear model $\mathbf{C}, \mathbf{D}$ of that sensor. The estimator is

$$
\hat{\dot{x}}=\mathbf{A} \hat{\boldsymbol{x}}+\mathbf{B} \boldsymbol{u}+\mathbf{L}\left(\boldsymbol{y}_{\mathrm{s}}-\mathbf{C} \hat{\boldsymbol{x}}-\mathbf{D} \boldsymbol{u}\right)
$$

where the feedback matrix $\mathbf{L}$ is found from a wellknown solution of the Ricatti equation, and is dependent on design matrices $\mathbf{Q}, \mathbf{R}$, and $\mathbf{S}$ (see, for example, reference [10]). Figure 1 shows the block diagram of the estimator.
In the optimal case, $\omega$ and $v$ are time-uncorrelated (white noise) processes with zero mean

$$
\begin{aligned}
& E\left(\boldsymbol{\omega}_{i} \boldsymbol{\omega}_{j}^{\mathrm{T}}\right)=0, \quad E\left(\boldsymbol{\omega}_{k}\right)=0 \\
& E\left(\boldsymbol{v}_{i} \boldsymbol{v}_{j}^{\mathrm{T}}\right)=0, \quad E\left(\boldsymbol{v}_{k}\right)=0
\end{aligned}
$$

where $E()$ is the expectation operator, applied to the errors at general $i, j, k$ time samples. The design matrices are the expected covariance of errors

$$
\mathbf{Q}=E\left(\boldsymbol{\omega}_{k} \boldsymbol{\omega}_{k}^{\mathrm{T}}\right), \quad \mathbf{R}=E\left(\boldsymbol{v}_{k} \boldsymbol{v}_{k}^{\mathrm{T}}\right), \quad \mathbf{S}=E\left(\boldsymbol{\omega}_{k} \boldsymbol{v}_{k}^{\mathrm{T}}\right)
$$

Note, however, that in practice the error sequences are usually difficult to determine, and frequently they are time correlated, so the components in $\mathbf{Q}$ and $\mathbf{R}$ are often set as approximates, or tuned to give best results. Critically, the $\mathbf{S}$ matrix is also commonly assumed to be zero - in many papers it is assumed to be unimportant, and it is frequently not even mentioned (e.g. references [3] and [6]).

It is tempting (and essentially valid) to think of the $\mathbf{Q}$ and $\mathbf{R}$ design matrices as a balance mechanism. In the case of low model error and high sensor error, the components in $\mathbf{R}$ should have high magnitude, with $\mathbf{Q}$ components relatively low. The expectation of high sensor errors results in low gains in $\mathbf{L}$, and in the extreme the filter tends to replicate the model; this is clear from equation (2), and Fig. 1, and the result is a correct design for an observer expected to operate under conditions of high sensor/low model error.

A similarly intelligent filter design for low sensor error/high model error combinations is not automatic, however; if the $\mathbf{S}=0$ assumption is used and the sensor set includes components with non-trivial

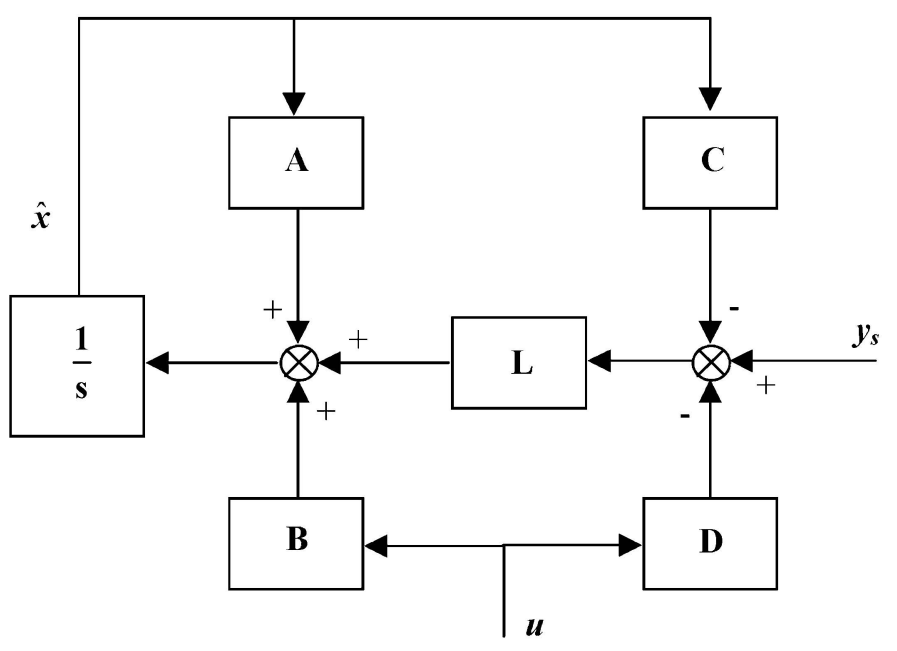

Fig. 1 Block diagram of the linear time-invariant Kalman filter 
sensor models $(\mathbf{C})$, the resulting filter can be very poor. This is well illustrated by the simple mechanical system of Fig. 2.

Consider a Kalman filter designed for this system, where the system matrices above are assumed to represent the true response. The observer is designed assuming that the model parameters have been poorly estimated

$$
\mathbf{A}=\left[\begin{array}{cc}
0 & 1 \\
-90 & -8
\end{array}\right], \quad \mathbf{C}=\left[\begin{array}{ll}
-90 & -8
\end{array}\right]
$$

This is an interesting proposition for two reasons. Not only does it induce high model error, it also illustrates the practical issue of using Kalman filters as observers for systems such as the vehicle; the error sequence will be time correlated, so condition $E\left(\omega_{i} \omega_{j}^{\mathrm{T}}\right)=0$ is not met and the resulting filter is strictly suboptimal. (The obvious subtext here is that, the more accurate the model and parameter estimates, the closer is the approach to a white noise model error process, and hence the closer is the approach to an optimal filter - this provides further motivation for the online parameter tuning facility of the filters designed later in this paper.)

To illustrate alternative designs, $\mathbf{Q}$ and $\mathbf{R}$, with two possibilities for $\mathbf{S}$, will be set as follows

$$
\begin{aligned}
& \mathbf{Q}=\left[\begin{array}{ll}
0 & 0 \\
0 & \alpha
\end{array}\right], \quad \mathbf{R}=\alpha+\varepsilon, {\left[\begin{array}{l}
0 \\
\alpha
\end{array}\right] \quad(\text { design 1) }} \\
& \mathbf{S}=\left[\begin{array}{l}
0 \\
0
\end{array}\right] \quad(\text { design 2) }
\end{aligned}
$$

Here, the top left component of $\mathbf{Q}$ is zero, so zero error is being assumed for $\omega(1)$; the equation for $\dot{x}(1)$ is known to be perfectly correct, as it comes from the state definitions; $\alpha$ represents the (mean square) magnitude of the acceleration error, which is caused by the poor parameter estimates in both $A$ and $C$.
This acceleration error appears as error processes $\omega(2)$ and $v$ and also as $E(\omega(2), v)$ in the (correct) crosscorrelation matrix for design $1 ; \varepsilon$ then represents some additional (white) noise on the accelerometer ( $\varepsilon$ must be non-zero to avoid rank deficiency in the Ricatti solution for design 1). Deliberately extreme settings $\alpha=1$ and $\varepsilon=10^{-8}$ are chosen to examine maximum reliance on sensor accuracy.

With design 1 the resulting filter can be written by reformulation of equation (2) as

$$
\hat{\hat{\boldsymbol{x}}}=\left[\begin{array}{cc}
-0.133 & 0.988 \\
-0.009 & -0.001
\end{array}\right] \hat{\boldsymbol{x}}+\left[\begin{array}{c}
-0.0015 \\
0.9999
\end{array}\right] \boldsymbol{y}_{\mathrm{s}}
$$

where the $\mathbf{L}$ matrix can be seen as the multiplier of $\boldsymbol{y}_{\mathrm{s}}$.

Note how the filter has achieved the desired sensor dependence here, with the velocity state estimated essentially from integration of the accelerometer, and the deflection from further integration of velocity. This is not true for design 2 , which yields

$$
\hat{\hat{\boldsymbol{x}}}=\left[\begin{array}{cc}
-2.92 & 0.741 \\
-127.3 & -11.3
\end{array}\right] \hat{\boldsymbol{x}}+\left[\begin{array}{c}
-0.0324 \\
-0.4142
\end{array}\right] \boldsymbol{y}_{\mathrm{s}}
$$

Here, without the cross-correlation information this filter simply advocates an even balance between the model and sensor information. It is also worth pointing out that the most obvious (if naive) assumption of high dynamic modelling error and low sensor error through design 3

$$
\mathbf{Q}=\left[\begin{array}{ll}
0 & 0 \\
0 & \alpha
\end{array}\right], \quad \mathbf{R}=\varepsilon, \quad \mathbf{S}=\left[\begin{array}{l}
0 \\
0
\end{array}\right]
$$

is even less effective, resulting in a very high gain

$$
\mathbf{L}=\left[\begin{array}{c}
-0.1 \\
-9999
\end{array}\right]
$$

Figure 3 illustrates these three filter designs by showing their response to a unit deflection initial

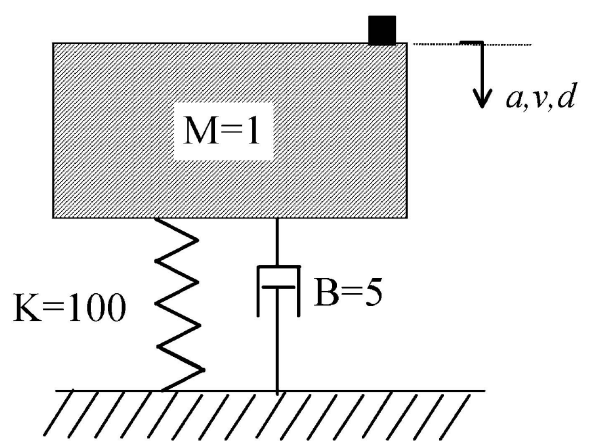

$$
\begin{gathered}
\underline{x} \equiv\left[\begin{array}{c}
\text { deflection } \\
\text { velocity }
\end{array}\right] \\
y_{s} \equiv[\text { acceleration }] \\
\underline{\dot{x}}=\left[\begin{array}{cc}
0 & 1 \\
-100 & -5
\end{array}\right] \underline{x} \\
y_{s}=\left[\begin{array}{ll}
-100 & -5
\end{array}\right] \underline{x}
\end{gathered}
$$

(zero inputs assumed for simplicity)

Fig. 2 Example system to illustrate Kalman filter design influences 

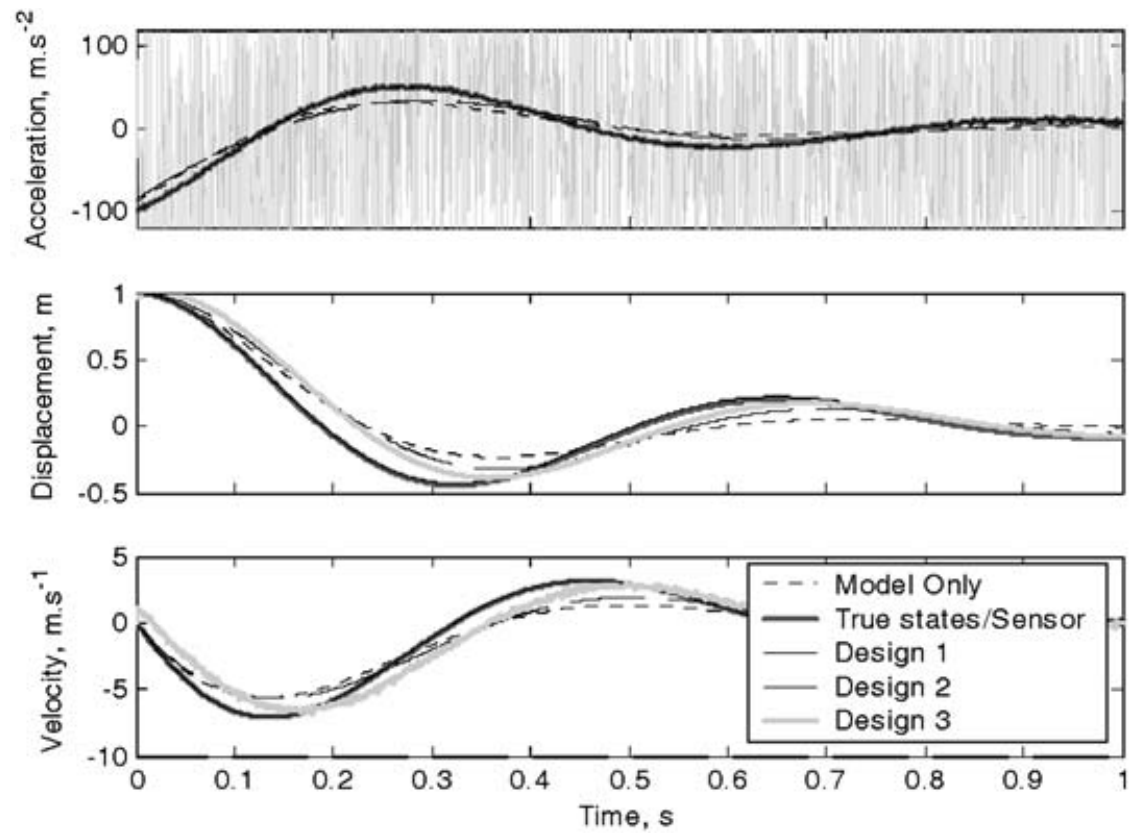

Fig. 3 State estimation from various Kalman filter designs

condition. The model error and high gain cause a huge magnification of the sensor noise within $\hat{y}$ in design 3 , and consequently high transmission of noise into $\hat{x}(2)$. The simplification of $\mathbf{S}$ results in a compromised result for design 2, and only design 1 achieves accurate tracking through correct use of the available sensor information.

These results are not intended to suggest that integration alone represents a good estimator design; clearly, any low-frequency sensor error would contradict such a claim. The important issue is rather that careful consideration should be given to proper inclusion of the cross-correlation matrix within any (inherently suboptimal) filter design of this type, in order to ensure that the best use is made of the instrumentation available.

\section{DESIGN OF AN IDENTIFYING KALMAN FILTER}

In order to design an adequate dynamic state observer, the Kalman filter model must be suitably realistic (e.g. non-linear/high order), yet, to achieve reliable online parameter identification and computational efficiency, a low-order model is preferable. The compromise here is to base the Kalman filter on a bicycle model (Fig. 4), yet incorporate non-linear, load-varying lateral tyre force characteristics.

Figure 4 depicts the bicycle handling model: $u$ is the forward velocity, $v$ is the lateral velocity, $r$ is the yaw velocity, $F_{y \mathrm{f}}$ is the total lateral force at the front

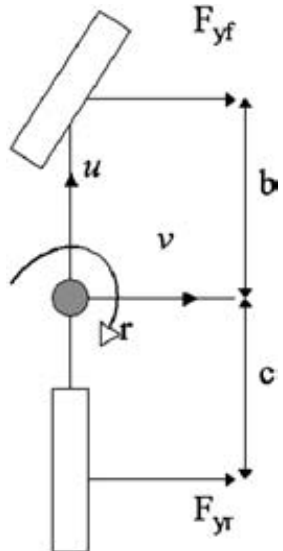

Fig. 4 Bicycle handling model

axle, $F_{y \mathrm{r}}$ is the total lateral force at the rear axle, $b$ is the distance from the front axle to the centre of gravity, and $c$ is the distance from the centre of gravity to the rear axle.

By inspection, the non-linear system model, $\boldsymbol{f}(x)$, is

$$
f(x)=\left[\begin{array}{c}
\dot{v} \\
\dot{r}
\end{array}\right]=\left[\begin{array}{c}
\frac{\sum_{i=1,4} F_{y i}}{M-u r} \\
\frac{\left(F_{y 1}+F_{y 2}\right) b-\left(F_{y 3}+F_{y 4}\right) c}{I_{z z}}
\end{array}\right]
$$

where, for each tyre, the lateral forces $F_{y i}$ are given by

$$
\begin{aligned}
& F_{y i}=F_{z i} D_{\mathrm{T}} \sin \left(C _ { \mathrm { T } } \operatorname { t a n } ^ { - 1 } \left\{B_{\mathrm{T}}\right.\right. {\left[\left(1-E_{\mathrm{T}}\right) \alpha+\left(E_{\mathrm{T}} / B_{\mathrm{T}}\right)\right.} \\
&\left.\left.\left.\times \tan ^{-1}\left(B_{\mathrm{T}} \alpha\right)\right]\right\}\right)
\end{aligned}
$$


This equation uses the simplest form of the Pacejka magic tyre formula [11] multiplied by the vertical load on each tyre, $F_{z i}$. Front/rear slip angles are given by

$$
\alpha_{\mathrm{f}}=\delta-\frac{v+b r}{u}, \quad \alpha_{\mathrm{r}}=-\frac{v-c r}{u}
$$

and vertical loads $F_{z i}$ are modelled as

$$
\begin{array}{ll}
F_{z 1}=\frac{c M g}{2(b+c)}+z_{\mathrm{f}}, & F_{z 2}=\frac{c M g}{2(b+c)}-z_{\mathrm{f}} \\
F_{z 3}=\frac{b M g}{2(b+c)}+z_{\mathrm{r}}, & F_{z 4}=\frac{b M g}{2(b+c)}-z_{\mathrm{r}}
\end{array}
$$

where the load transfer is calculated at each iteration of the Kalman filter by using the lateral forces from the previous time step [denoted by superscript $(-)$ ]

$$
z_{\mathrm{f}}=\frac{h\left(F_{y 1}^{(-)}+F_{y 2}^{(-)}\right)}{t_{\mathrm{f}}}, \quad z_{\mathrm{r}}=\frac{h\left(F_{y 3}^{(-)}+F_{y 4}^{(-)}\right.}{t_{\mathrm{r}}}
$$

Now, the state vector is to be augmented by system parameters, $\boldsymbol{\theta}$, that are suitable for identification. Avoiding those that are either well known, or that have limited dynamic influence, the focus here will be upon tyre parameters (two cases are considered, $\boldsymbol{\theta}=D_{\mathrm{T}}$ and, later, $\left.\boldsymbol{\theta}=\left[C_{\mathrm{T}}, D_{\mathrm{T}}\right]\right)$. As there is no model for the expected variation of these parameters, the expanded state/parameter vector is modelled as

$$
\boldsymbol{f}(x)=\left[\begin{array}{c}
\dot{v} \\
\dot{r} \\
\dot{\theta}
\end{array}\right]=\left[\begin{array}{c}
\frac{\sum_{i=1,4} F_{y i}}{M-u r} \\
\frac{\left(F_{y 1}+F_{y 2}\right) b-\left(F_{y 3}+F_{y 4}\right) c}{I_{z z}} \\
0
\end{array}\right]
$$

The sensors are mass centre lateral acceleration and yaw rate

$$
\boldsymbol{h}(x)=\left[\begin{array}{c}
\dot{v}+u r \\
r
\end{array}\right]
$$

and the parameters, where not identified, are fixed according to Table 1 . In addition to the steer angle input $\delta$, this model also treats the vehicle forward speed $u$ as an input. In the simulation exercise this is assumed to be known from the source model, and on the test vehicle it is estimated by summation of the two undriven (rear) wheel speed signals, appropriately compensated through an estimate of the wheel rolling radius.
Table 1 Vehicle parameters

\begin{tabular}{ll}
\hline Parameter & Quantity \\
\hline$M$ & $1700 \mathrm{~kg}$ \\
$I_{z z}$ & $1800 \mathrm{~kg} \mathrm{~m}{ }^{2}$ \\
$b$ & $1.1667 \mathrm{~m}$ \\
$c$ & $1.333 \mathrm{~m}$ \\
$t_{\mathrm{f}}$ & $1.4 \mathrm{~m}$ \\
$t_{\mathrm{r}}$ & $1.5 \mathrm{~m}$ \\
$h$ & $0.25 \mathrm{~m}$ \\
$\mu$ & 0.9 \\
$B_{\mathrm{T}}$ & 9 \\
$C_{\mathrm{T}}$ & 0.927 \\
$D_{\mathrm{T}}$ & 1.06 \\
$E_{\mathrm{T}}$ & 0.5 \\
\hline
\end{tabular}

The non-linear, or extended, Kalman filter (EKF) from references [9] and [10] employs system and sensor Jacobians to determine, to a first approximation, the migration sensitivity of the state vector

$$
\begin{aligned}
& \mathbf{F}(\hat{x}(t))=\left.\frac{\partial f(x(t))}{\partial x(t)}\right|_{x(t)-\hat{x}(t)} \\
& \mathbf{H}(\hat{x}(t))=\left.\frac{\partial h(x(t))}{\partial x(t)}\right|_{x(t)=\hat{x}(t)}
\end{aligned}
$$

and the filter design is fully determined by recursive calculation of the Ricatti matrix

$$
\begin{aligned}
& \mathbf{L}_{k}= \mathbf{P}_{k} \mathbf{H}^{\mathrm{T}}\left(\hat{\boldsymbol{x}}_{k}\right)\left[\mathbf{H}\left(\hat{\boldsymbol{x}}_{k}\right) \mathbf{P}_{k} \mathbf{H}^{\mathrm{T}}\left(\hat{\boldsymbol{x}}_{k}\right)+\mathbf{R}\right]^{-1} \\
& \mathbf{P}_{k}^{*}=\left[\mathbf{I}-\mathbf{L}_{k} \mathbf{H}\left(\hat{\boldsymbol{x}}_{k}\right)\right] \mathbf{P}_{k} \\
& \mathbf{P}_{k+1}=\mathbf{P}_{k}^{*}+\int\left(\mathbf{F}^{*}\left(\hat{\boldsymbol{x}}_{k}\right) \mathbf{P}_{k}^{*}+\mathbf{P}_{k}^{*} \mathbf{F}^{*}\left(\hat{\boldsymbol{x}}_{k}\right)+\mathbf{Q}^{*}\right) \mathrm{d} t \\
& \hat{\boldsymbol{x}}_{k+1}=\hat{\boldsymbol{x}}_{k}+\mathbf{L}_{k}\left(\boldsymbol{y}_{k}-\boldsymbol{h}\left(\hat{\boldsymbol{x}}_{k}\right)\right) \\
& \quad+\int\left(\boldsymbol{f}\left(\hat{\boldsymbol{x}}_{k}\right)+\mathbf{S R}^{-1}\left(\boldsymbol{y}_{k}-\boldsymbol{h}\left(\hat{\boldsymbol{x}}_{k}\right)\right)\right) \mathrm{d} t
\end{aligned}
$$

where

$$
\mathbf{F}^{*}\left(\hat{\boldsymbol{x}}_{k}\right)=\mathbf{F}\left(\hat{\boldsymbol{x}}_{k}\right)-\mathbf{S R}^{-1} \mathbf{H}\left(\hat{\boldsymbol{x}}_{k}\right), \quad \mathbf{Q}^{*}=\mathbf{Q}-\mathbf{S R}^{-1} \mathbf{S}^{\mathbf{T}}
$$

A sampling interval of $200 \mathrm{~Hz}$ is used, and the integral over each time step is conducted using the fourthorder Runge-Kutta method; both of these precautions are taken to ensure stability under the rapid parameter estimation that is desired.

It is worth noting that the Jacobian equations (16) are formulated analytically here, so that all state/ state and state/parameter sensitivities are properly modelled, although these are zero order held within each Runge-Kutta time step owing to their dependence on the time-lagged lateral load transfer.

Finally, the $\mathbf{Q}, \mathbf{R}$, and $\mathbf{S}$ noise balance matrices are determined from a simulation exercise. As it would 
be impossible to quantify these matrices precisely for a test vehicle, a sequence of $\omega_{k}$ and $v_{k}$ is established by comparing the Kalman filter model derived above with simulation output from a higher-order model, having in this case a combined slip tyre model, roll degree of freedom, and longitudinal weight transfer. Noise estimates thus come from

$$
\boldsymbol{\omega}_{k}=\left[\begin{array}{c}
\bar{v} \\
\overline{\dot{r}} \\
\overline{\dot{\theta}}
\end{array}\right]-\boldsymbol{f}(v, r, \theta), \quad \boldsymbol{v}_{k}=\left[\begin{array}{c}
\bar{v}-u \bar{r} \\
\bar{r}
\end{array}\right]-\boldsymbol{h}(v, r, \theta)
$$

where $\overline{\dot{v}}, \bar{r}$, etc., are the higher-order simulation states and $\boldsymbol{f}$ and $\boldsymbol{h}$ are the Kalman filter designs outlined above. The precise simulation input here is not critical, but some dynamic variation must also be made in the parameters to be identified, to establish correlations between state and parameter errors due to their variation.

Matrices $\mathbf{Q}, \mathbf{R}$, and $\mathbf{S}$ are constructed from covariance matrices, with the parameter identification moderated using a sensitivity value $\lambda$ to regulate their rate of adaptation

$$
\begin{aligned}
& \mathbf{Q}=\mathbf{I}_{\lambda} \operatorname{cov}\left(\boldsymbol{\omega}_{k}\right) \mathbf{I}_{\lambda} \\
& \mathbf{S}=\mathbf{I}_{\lambda} \operatorname{cov}\left(\boldsymbol{v}_{k}, \boldsymbol{\omega}_{k}\right), \\
& \mathbf{R}=\operatorname{cov}\left(\boldsymbol{v}_{k}\right)
\end{aligned} \quad \mathbf{I}_{\lambda}=\left[\begin{array}{llll}
1 & 0 & 0 & 0 \\
0 & 1 & 0 & 0 \\
0 & 0 & \lambda & 0 \\
0 & 0 & 0 & \lambda
\end{array}\right]
$$
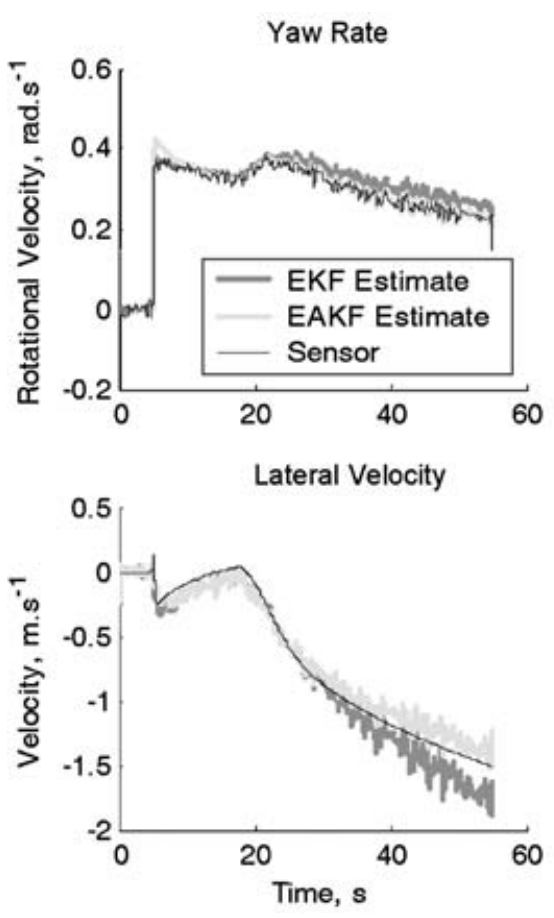

\section{SIMULATION EXPERIMENT}

The proposed Kalman filter designs are tested with and without parameter identification. These will be referred to as the (standard) extended Kalman filter (EKF) and (adaptive) identifying Kalman filter (IKF).

The higher-order model referred to in section 3 was simulated over a $3^{\circ}$ step steer event at $t=5 \mathrm{~s}$ at an initial vehicle speed $u=20 \mathrm{~m} / \mathrm{s}$, with an additional step change applied to the wheel torque from 5 to $300 \mathrm{~N} \mathrm{~m}$ at $t=18 \mathrm{~s}$. Independent white noise sources were added to the simulated sensors, with r.m.s. values of $2 \mathrm{~m} / \mathrm{s}^{2}$ and $0.05 \mathrm{rad} / \mathrm{s}$ on the lateral acceleration and yaw rate respectively; for consistency, these magnitudes are similar to those experienced within the vehicle test experiment in section 5 and are in sympathy with the noise levels applied in the noise matrix design process.

The IKF was formulated to vary only the magnitude of the tyre force, parameter $D_{\mathrm{T}}$, with its value initialized slightly higher than default in order to examine its adaptation to the steer input. The design parameter $\lambda$ was set to $10^{-5}$, to ensure a suitably rapid, yet noise-free adaptation of the parameter; higher settings invoke a faster but more noisy estimation, and extreme settings can cause instability.

Figure 5 shows the simulated sensors and their filtered estimates, along with the remaining state estimate, lateral velocity, and the estimated $D_{\mathrm{T}}$ parameter for the IKF. The initially high value of $D_{\mathrm{T}}$ causes
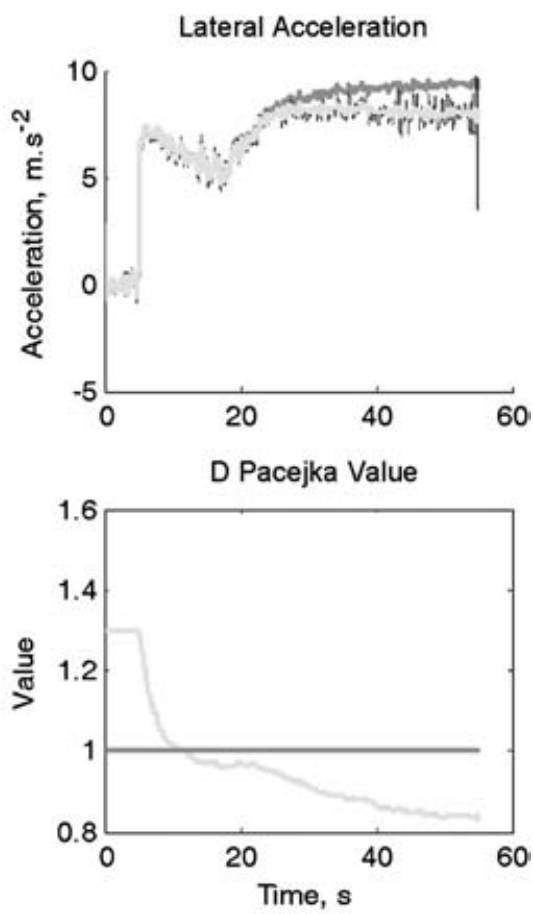

Fig. 5 Filtered sensors, inputs, and adapted tyre parameters 
an overshoot in estimation of the yaw rate at $5 \mathrm{~s}$, but the adapted parameter then settles to a value close to the default value (set in the EKF) before the torque at the wheels is changed. Up to this point, the EKF and the IKF converge to produce similar results, as would be expected given the convergence of the tyre parameter. Around $25 \mathrm{~s}$ the EKF becomes less effective at filtering the sensors, however, and develops a steady state error. Meanwhile, the IKF maintains good noise-filtering capabilities, with the adapted parameter allowing accurate low-frequency tracking.

Figure 6 shows the actual and estimated slip angles and forces for the front and rear axles, with similar trends in the results. The test is interesting in that the Kalman filter model does not include any terms to explain the loss of lateral force capability that comes about owing to the increase in torque. The IKF is able to compensate for the effective saturation of the tyre that the torque step induces through the combined slip tyre in the source model, by lowering the $D_{\mathrm{T}}$ parameter. This results in more accurate state estimations, but also consequently improves the accuracy of intermediate variables within the Kalman filter model, such as the tyre forces.

\section{VEHICLE EXPERIMENT}

The IKF is further examined on sensor readings from a test vehicle (Ford Mondeo) performing a variety of manoeuvres at a proving ground. Now two Pacejka shape parameters, $C_{\mathrm{T}}$ and $D_{\mathrm{T}}$, are simultaneously adapted on the front axle. This will give more flexibility to the Kalman filter to alter the shape of the tyre curve to suit changes in drive torque, friction, and temperature that occur during the course of each test. The noise matrices are the same as used on the simulation experiment, since the sensor noise levels have been matched. Also the same adaptation rate, $\lambda$, is used now for both shaping parameters.

Figure 7 has the results from a step steer test conducted at $u=14.7 \mathrm{~m} / \mathrm{s}$, showing successful filtering of sensors, and adaptation of the tyre curve map, along with the estimated tyre force. The initial tyre shaping parameters were deliberately set higher than expected, and it can again be seen that the tyre curve adapts quickly to the change in vehicle state. The tyre curve estimation could provide useful information for a control system to calculate the best potential course of action when approaching a limit handling situation.

Figure 8 shows the same variables, but under a different test condition; here a section of data is taken from a test drive on a handling circuit, showing a series of steering fluctuations. The same trends are visible as in the graph above, with successful filtering and accurate tracking of the sensor data allied to rapid modulation of the tyre curve. Again, a low noise estimation of the tyre forces is apparent.

Finally, Fig. 9 shows estimates of the slip angles and corresponding tyre forces at the front and rear. The vertical loads that the IKF generates as
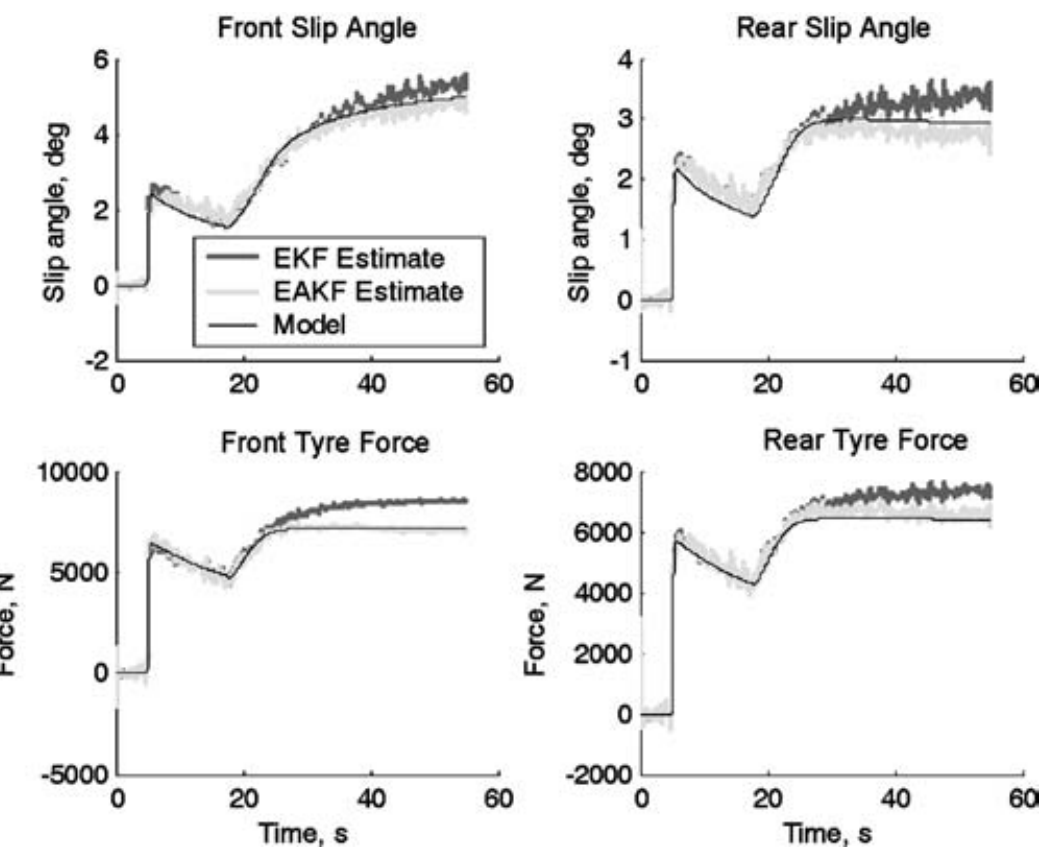

Fig. 6 Tyre slip angles and forces 

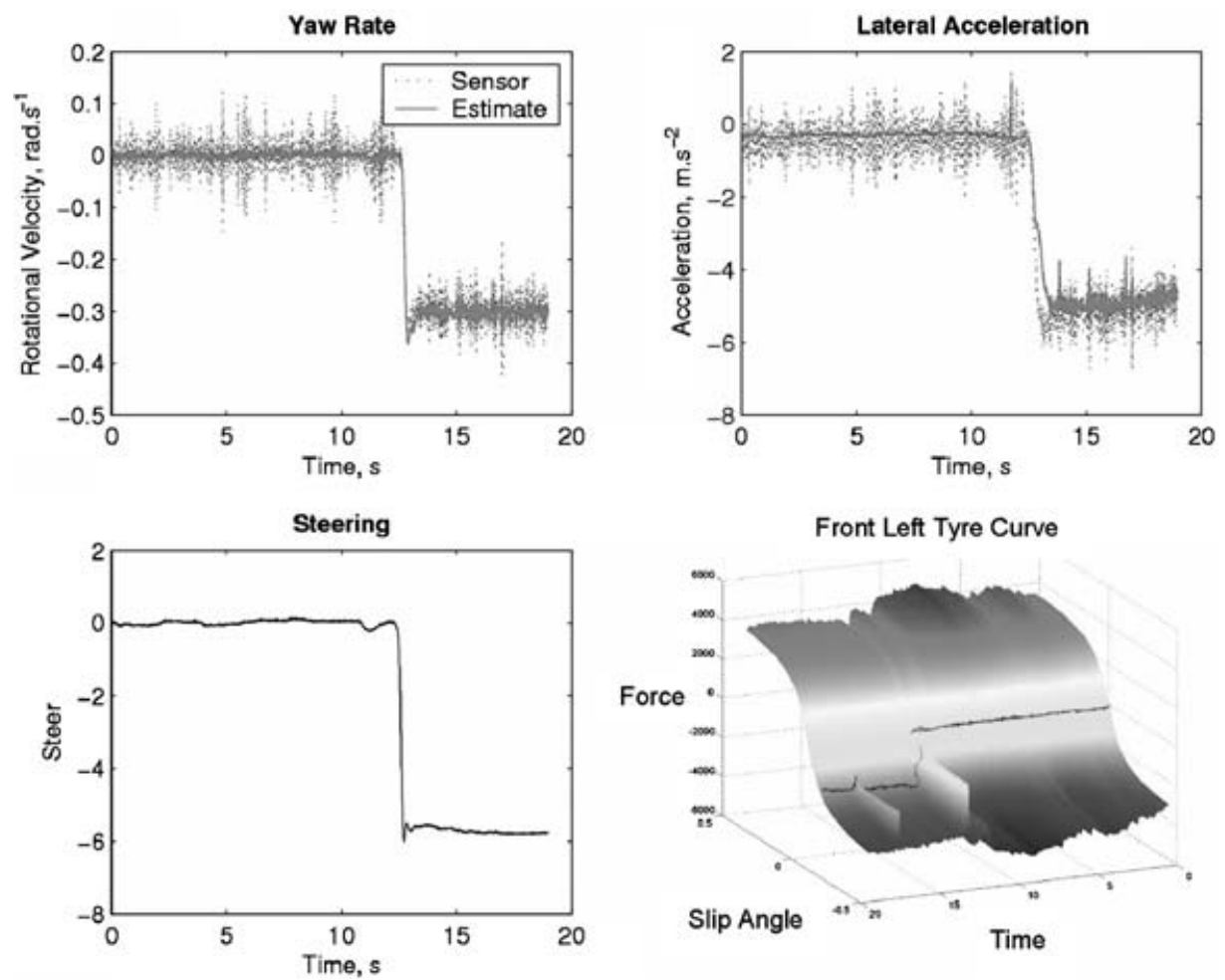

Fig. 7 Steering input, filtered sensor data, and adapted tyre curve for step steer
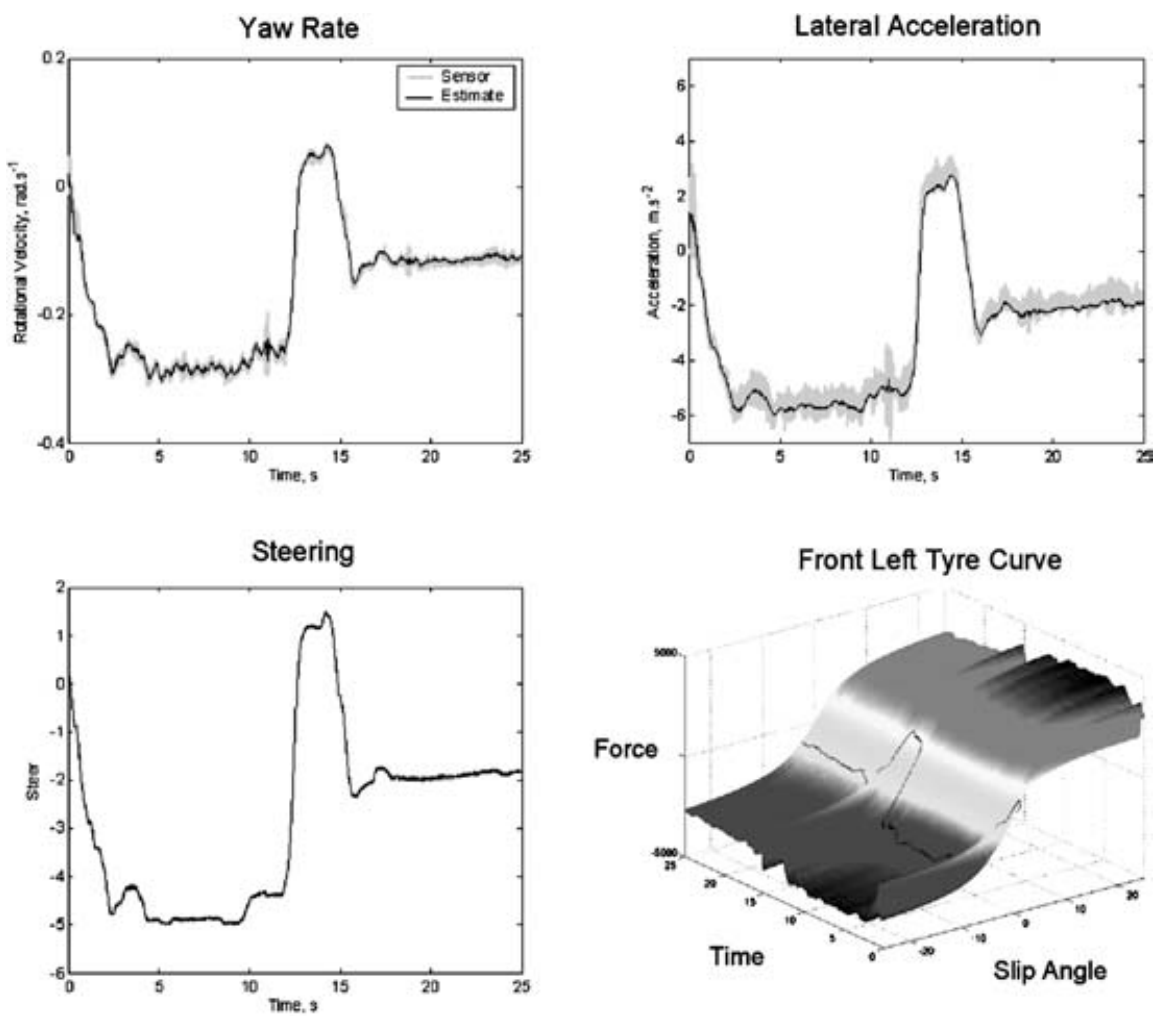

Fig. 8 Steering input, filtered sensor data, and adapted tyre curve for a section of test data 

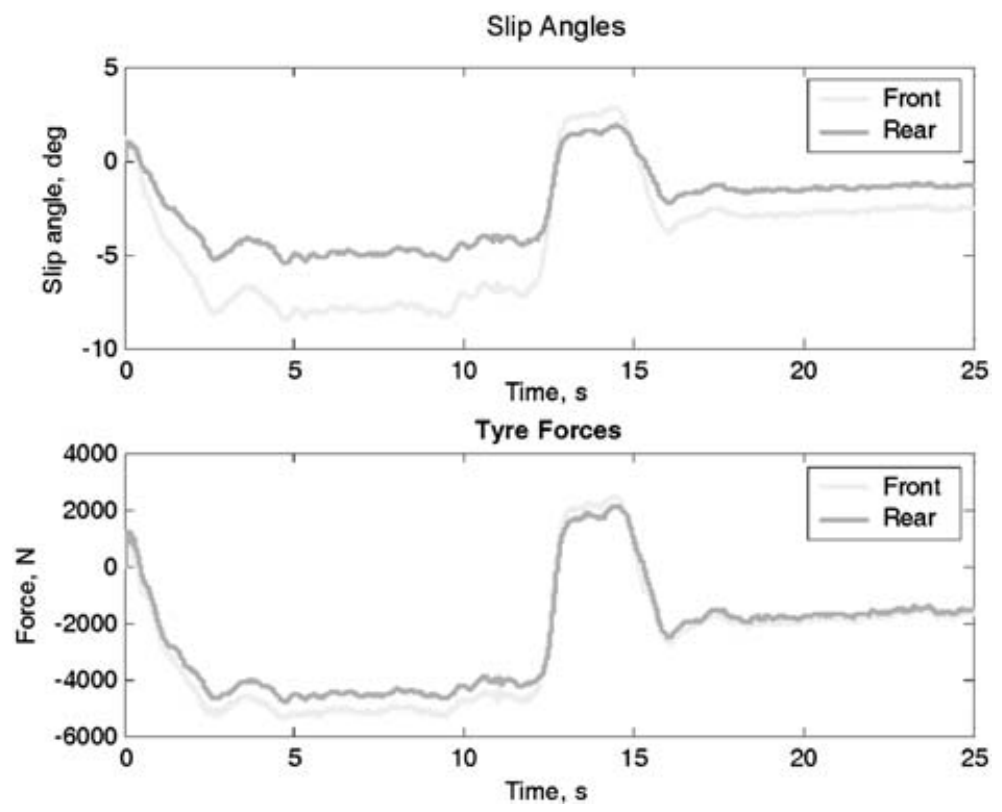

Fig. 9 Slip angles and lateral force estimates

intermediate variables in the state estimation process is illustrated in Fig. 10.

The combination of a simple, yet non-linear model structure within the observer, allied with tyre model identification, provides great potential for future development of Kalman filter based observers that can provide the full variety of real-time vehicle information required in modern vehicle diagnostic and control systems.

\section{CONCLUDING REMARKS}

This paper has demonstrated the potential for combined state and parameter estimation, with a view to expanding real-time information about the vehicle, particularly with respect to tyre forces. It also illustrates the importance of good design practice for all Kalman filters, in terms of the effect of the noise cross-correlation matrix. The identifying Kalman filter

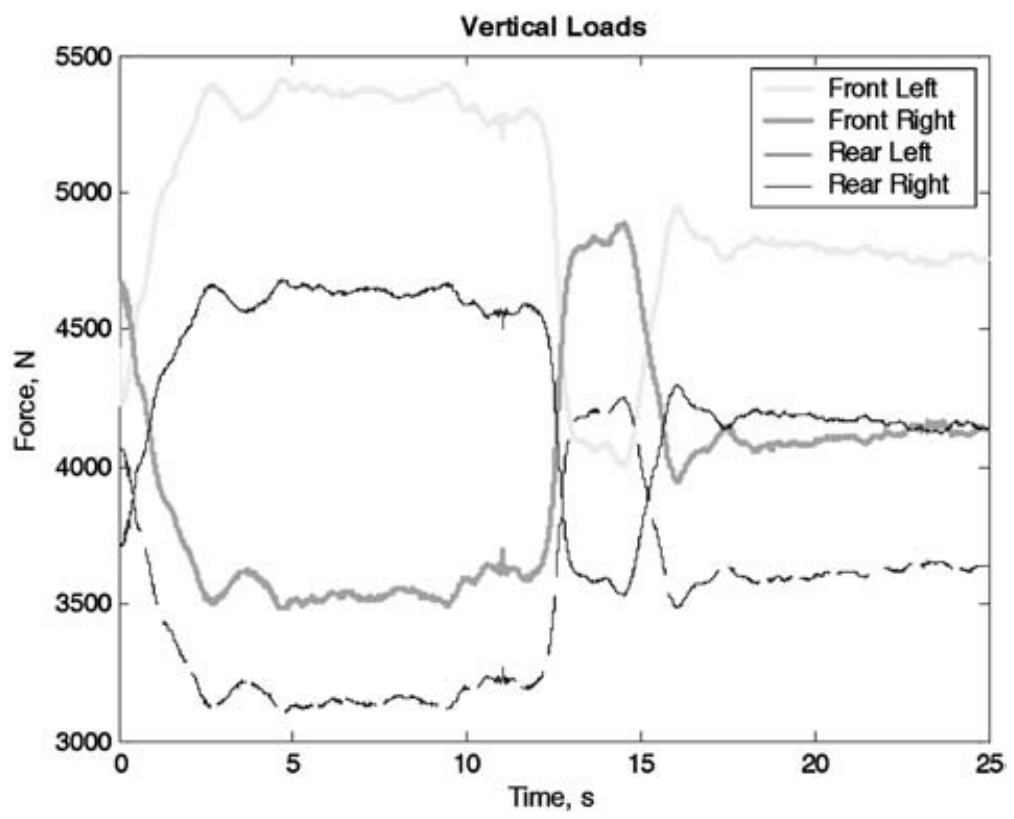

Fig. 10 Vertical loads from a section of test data 
has been shown to operate successfully in simulation and on a test vehicle over a variety of tests.

There is every reason to suggest that filters of this type can be successfully implemented, in real time, on production vehicles. Also, there is scope for the integration of further (e.g. ride) modes, provided these are sufficiently distinct in their dynamic influence. For example, a mass-adaptive heave/pitch mode could readily be added to the rigid body vehicle model discussed here, providing suspension state information and also compensating for mass and consequential yaw inertia variability in both ride and handling behaviour. There is, however, also the need to be cautious in the selection of parameters to be identified online, since underdetermination of the dynamic states can result.

\section{REFERENCES}

1 Ray, L. Nonlinear tire force estimation and road friction identification: simulation and experiments. Automatica, 1997, 33(10).

2 Ray, L. Nonlinear tire force estimation and road friction identification: field test results. SAE paper 960181, 1996.

3 Zuurbier, J. and Bremmer, P. State estimation for integrated vehicle dynamics control. Proceedings of AVEC 2002.

4 Bevly, D. M., Gerdes, J. C., and Wilson, C. The use of GPS based velocity measurements for measurement of sideslip and wheel slip. Veh. Syst. Dynamics, 2002, 38(2).

5 Ryu, J., Rossetter, E. J., and Gerdes, J. C. Vehicle sideslip and roll parameter estimation using GPS. Proceedings of AVEC 2002.

6 Carlson, C. R., Gerdes, J. C., and Powell, J. D. Practical position and yaw rate estimation with GPS and differential wheelspeeds. Proceedings of AVEC 2002.

7 Fukuba, H., Adachi, T., Yoshimoto, A., Takahashi, H., and Yoshioka, T. Precise 6-DOF movement measurement for vehicle by GPS and angular rate sensors and Kalman filter. Proceedings of AVEC 2002.

8 Huh, K., Kim, J., and Yi, K. Monitoring system design for estimating the lateral tyre force. Proc. IMechE, Part D: J. Automobile Engineering, 2003, 217.

9 Best, M., Gordon, T., and Dixon, P. An extended adaptive Kalman filter for real time state estimation of vehicle handling dynamics. Veh. Syst. Dynamics, 2000, 34(1).

10 Gelb, A. Applied optimal estimation, 1986 (MIT Press, Cambridge, Massachusetts).

11 Pacejka, H. B., Bakker, E., and Lidner, L. A new tire model with an application in vehicle dynamic studies. SAE paper 890087, 1989.

\section{APPENDIX}

Notation

Dynamic variables

$F_{y f}$

$F_{\text {yr }}$

$r$

$u$

v

$\boldsymbol{x}$

$\dot{\boldsymbol{x}}$

$\hat{\boldsymbol{x}}$

$y$

$\hat{y}$

$\delta$

front axle lateral force $(\mathrm{N})$

rear axle lateral force $(\mathrm{N})$

yaw rate $(\mathrm{rad} / \mathrm{s})$

longitudinal velocity $(\mathrm{m} / \mathrm{s})$

lateral velocity $(\mathrm{m} / \mathrm{s})$

state vector

state derivative vector

estimate of state vector

sensor set

estimate of sensor set

front wheel steer angle (rad)

\section{Model parameters}

$b$

$B_{\mathrm{T}}, C_{\mathrm{T}}, D_{\mathrm{T}}, E_{\mathrm{T}}$

c

$g$

$h$

$I_{z z}$

$M$

$t_{\mathrm{f}}, t_{\mathrm{r}}$

$\mu$

Kalman filter

$\boldsymbol{f}(x)$

$\mathbf{F}(x)$

$\boldsymbol{h}(x)$

$\mathbf{H}(x)$

$\mathbf{L}$

$\mathbf{P}$

$\mathbf{Q}$

$\mathbf{R}$

S

v centre of gravity (CG) to the front axle

Pacejka tyre curve shape

parameters

CG to the rear axle

gravitational constant

height of CG above ground

yaw moment of inertia

vehicle mass

front and rear track respectively

tyre friction coefficient

non-linear system model

system model Jacobian

non-linear output model

output model Jacobian

optimal gain matrix

state error covariance matrix

process noise covariance matrix

measurement noise covariance

matrix

measurement/process cross-

covariance matrix

measurement noise (sensor error) process

process noise (state error) process 